\title{
Phase II Study of Post-surgery Radiotherapy Combined with Recombinant Adeno-viral Human P53 Gene Therapy in Treatment of Oral Cancer
}

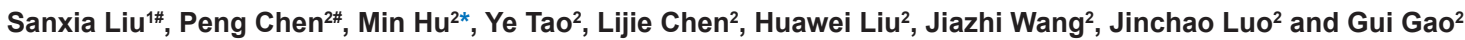

${ }^{1}$ Department of Stomatology, Nankai University of Medicine, Tianjin 300071, China

${ }^{2}$ Department of Stomatology, 301 Military General Hospital, Beijing 100853, China

"Co-first authors

\begin{abstract}
Objective: To evaluate benefits of Recombinant Adeno-viral Human p53 (rAd-p53) gene therapy combined with radiotherapy in prevention of oral cancer recurrence after a radical resection.

Methods: A total of 215 patients with resectable Tongue Cancer (TCa) and 268 patients with resectable Gingival Carcinoma (GCa) satisfying the inclusion criteria and were randomly assigned to two groups: the Experiment Group (EG) and the Control Group (CG). The EG received multi-point injections of rAd-p53 into the wound surface at a dose of $1 \times 10^{12}$ Viral Particles (VP) after a radical resection. Both $E G$ and $C G$ were given radiotherapy at a total dose of 60 Gy three weeks after surgery. All these patients will be followed at least for 3 years.

Results: Among these 483 cases, 107 patients (57 in EG and 50 in CG) finished 3-years follow-up. Two cases (2/27) of TCa and $2(2 / 30)$ in GCa patients had a local recurrence in EG, but $8(8 / 24)$ TCa and $8(8 / 26)$ GCa patients in CG had a local recurrence. Both recurrent rates of $\mathrm{TCa}(33.3 \%)$ and $\mathrm{GCa}(30.8 \%)$ in $\mathrm{CG}$ are statistically significantly higher than that of TCa $(7.4 \%)$ and $\mathrm{GCa}$ $(6.7 \%)$ in $E G$, respectively. The overall recurrent rate in EG is $7.0 \%$, which is also statistically significantly lower than that $(32 \%)$ in CG. Overall 3-years survival (OS) rate of EG is $100 \%$ and the progress free survival (PFS) rate is $93.0 \%$ and the minimum PFS time is 29 months. The 3-years OS and PFS rates of CG are $94.0 \%$ and $68.0 \%$, respectively. Except for self-limited fever, no other adverse reaction was found to be relative to rAd-p53.
\end{abstract}

Conclusions: Post-tumorectomy wound surface injection of rAd-p53 combining with radiotherapy is safe, and may prevent local recurrence and increase both OS and PFS rate for the patients with TCa or GCa.

Keywords: Recombinant adeno-viral human p53; Gene therapy; Oral cancer

\section{Introduction}

P53 gene has been well studied and is one of most important tumor suppressing gene. It has multiple anti-tumor functions including blocking cell cycle, inducing cell apoptosis, inhibiting tumor angiogenesis and sensitizing tumor to chemo- and radio-therapy. The p53 mutation occurs up to $63 \%$ of oral squamous carcinoma $[1,2]$. Loss of p53 normal functions is associated with tumorigenesis, chemoand radio-therapy resistance $[3,4]$. Gendicine, the first antitumor gene product registered for marketing in the world, is a recombinant adenoviral p53 gene (rAd-p53), in which, a wild type p53 gene was inserted in a replication-defective adenovirus type 5. A phase II clinical trial rAd-p53 intratumoral injection combined with radiotherapy in treatment of patients with advanced head and neck cancer showed the combined therapy could significantly increase the 5-years Overall Survival (OS) and Progress Free Survival (PFS) rates and prolong OS and PFS [5]. Several reports demonstrated rAd-p53 was effective for lung, liver, breast, and cervical cancers in advanced stage [6-10].

There are several types of oral cancers, but around $90 \%$ are squamous cell carcinomas. Overall, 9-12\% adults per 100,000 will develop oral cancer. Most oral cancer occurs in those over the age of 40 and its rates increase with age. The increase becomes more rapid after age 50 and peaks between ages 60 and 70 . The main treatments for patients with oral cancer include surgery and radiotherapy. Chemotherapy is also used to treat oral cancer, but it is usually used in patients with metastasis.

Although great improvements have been made in treatment of oral squamous carcinoma using combination of traditional anti-cancer treatments, the 5-years overall survival rate is still kept around $60 \%$, and local recurrence is the main reason of treatment failure. Here, we reported using post-surgery p53 gene therapy combined with radiotherapy can reduce the local recurrence rate and increase survival time.

\section{Methods}

The study was proved by our hospital ethics committee but not registered in an international clinical trial registry platform. All the enrolled patients signed a consent form.

\section{Patients}

From January 2005 to April 2009, a total of 215 patients with resectable Tongue Cancer (TCa) and 268 patients with resectable Gingival Carcinoma (GCa) satisfying the inclusion criteria and were randomly assigned to two groups: the Experiment Group (EG) and the Control Group (CG). Among these patients, 107 cases have finished 3-years follow-up and are included in this reports: 57 in EG including $27 \mathrm{TCa}$ and $30 \mathrm{GCa}$ patients and 50 in CG including $24 \mathrm{TCa}$ and 26 GCa patients. The inclusion criteria are: histopathologically diagnosed resectable TCa or GCa; over 18 years old; with an Eastern Cooperative Oncology Group (ECOG) score of 0-2; with normal hemogram, blood coagulation, liver and kidney function tests; understanding and signing

*Corresponding author: Min Hu, Department of Stomatology, 301 Military Genera Hospital, Beijing 100853, China, E-mail: humin301mdph@163.com

Received May 23, 2012; Accepted July 07, 2012; Published July 09, 2012

Citation: Liu S, Chen P, Hu M, Tao Y, Chen L, et al. (2012) Phase II Study of Post-surgery Radiotherapy Combined with Recombinant Adeno-viral Human P53 Gene Therapy in Treatment of Oral Cancer. J Cancer Sci Ther 4: 193-195. doi:10.4172/1948-5956.1000140

Copyright: (c) 2012 Liu S, et al. This is an open-access article distributed unde the terms of the Creative Commons Attribution License, which permits unrestricted use, distribution, and reproduction in any medium, provided the original author and source are credited. 
the informed consent form. The patients' characteristics and disease stage are summarized in Table 1 by the treatment group.

\section{Treatments}

RAd-p53 (brand name is Gendicine ${ }^{\circledR}$ ) was purchased from Shenzhen Sibiono Genetech Co. Ltd. The patients in EG received the wound surface injection of rAd-p53 at a dose of $1 \times 10^{12}$ Viral Particles (VP) after a radical resection of tumor and radiotherapy three weeks after the surgery. RAd-p53 was diluted into $5 \mathrm{ml}$ of physiological saline and then was injected into the wound surface at multiple points. The patients in CG only received radiotherapy three weeks after surgery. Radiotherapy was given 5 times per week for 6 weeks for a total dose of $60 \mathrm{~Gy}$. All these patients were followed up every 3 month. Routine physical examination, lab tests including hemogram, liver and kidney function tests, and morphologic imaging tests were performed during each follow-up.

\section{Results}

Among these 483 cases, 107 patients (57 in EG and 50 in CG) finished 3-years follow-up. Two (2/27) of TCa and 2 (2/30) of GCa patients in EG had a local recurrence, but $8(8 / 24)$ of TCa and $8(8 / 26)$ of GCa patients in CG had a local recurrence. Both recurrent rates of TCa (33.3\%) and GCa (30.8\%) in CG are statistically significantly higher than that of $\mathrm{TCa}(7.4 \%)$ and $\mathrm{GCa}(6.7 \%)$ in $\mathrm{EG}$, respectively. The overall recurrent rate in EG is $7.0 \%$, which is also statistically significantly lower than that (32\%) in CG. Overall 3-years survival rate of EG is $100 \%$ and the progress free survival (PFS) rate is $93.0 \%$ and the minimum PFS time is 29 months. The 3 -years OS and PFS rates of CG are $94.0 \%$ and $68.0 \%$, respectively. The following Figure 1 shows the recurrent rates of TCa and GCa in EG and CG, respectively.

Except for self-limited fever, no other Adverse Effect (AE) was found to be relative to rAd-p53. Forty-eight patients (84.2\%) in EG had fever of $37.5-39.2^{\circ} \mathrm{C}$, comparing to $12 \%$ of the patients in CG with of fever of $37.5-38^{\circ} \mathrm{C}$. The fever lasted $2-12$ hours and reduced to normal without any treatment, or by physical cooling. The AEs considered to be relative to radiotherapy included oral and pharyngeal pain, dysphagia, xerosis cutis, desquamation, skin or oral membrane burn and xerostomia.

\section{Discussions}

Wild type p53 gene plays an important role in regulation of cell cycle and apoptosis and suppresses tumor growth [11]. Normal functional p53 protein is associated with preventing tumorogenesis, inhibiting tumor growth and metastasis, increasing tumor sensitivity to radiotherapy and chemotherapy [12]. The mutant forms of the p53 protein have lost these tumor suppressor activities. Some mutant forms of p53 may gain new functions and interfere with cell growth suppressor effects of wild-type p53. Several previous studies showed introduction of wild type p53 gene into tumor cells recovered the

\begin{tabular}{|l|l|l|}
\hline Characteristics & EG & CG \\
\hline Age (years old) & $57.2 \pm 22.4(31-80)$ & $56.6 \pm 23.7(27-76)$ \\
\hline $\begin{array}{l}\text { Gender } \\
\text { Male }\end{array}$ & \\
Female & $35(61.4 \%)$ & $26(52.0 \%)$ \\
\hline Tissue Type & $22(38.6 \%)$ & $24(48.0 \%)$ \\
TCa & $27(47.4 \%)$ & $24(48.0 \%)$ \\
GCa & $30(52.6 \%)$ & $26(52.0 \%)$ \\
\hline
\end{tabular}

Table 1: Patients' characteristics.

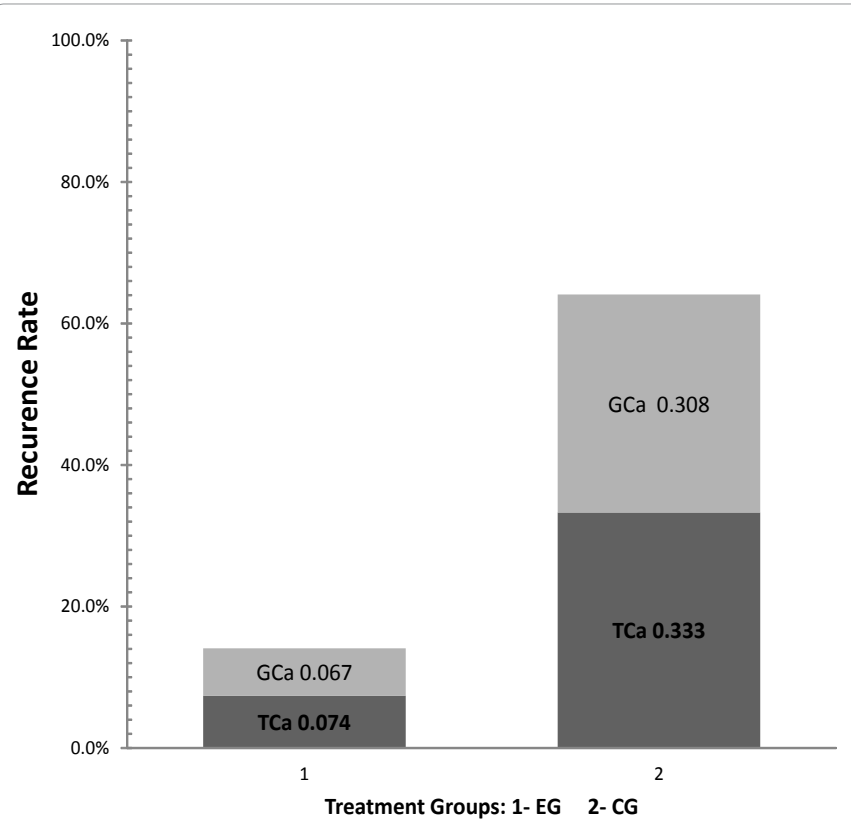

Figure 1: Recurrect rate of TCa and GCa in EG and CG.

anti-tumor activities of $\mathrm{p} 53$ and also regain tumor sensitivity to chemotherapy and radiotherapy [13-15].

P53 gene delivered using adenovirus-based vector has been demonstrated to be effective and safe in treatment of several types of malignant tumors. A phase II clinical trial using rAd-p53 intratumor injection combined with radiotherapy in treatment of patients with advanced head and neck cancer showed that p53 gene therapy combined with radiotherapy significantly increased complete response rate, overall response rate, the 5-years OS and PFS rates, and prolonged OS and PFS comparing to radiotherapy alone [5]. Several reports demonstrated rAd-p53 is effective for lung, liver, breast, cervical cancers, and some pre-cancerous lesions such as oral leukoplakia and cervical displasia. In these clinical studies, p53 gene therapy was combined with chemotherapy, radiotherapy, or chemoand radiotherapy. In treatment of liver cancer, the gene therapy was often used with transcatheter arterial chemoembolization. P53 gene therapy was also used in combination with surgery: before, during or after a surgery. The surgical wound injection of rAd-p53 has been used for preventing local recurrence of malignant gliomas, colon and rectal cancer, and cervical cancer [unpublished data]. So far, there has been no sufficient data to prove the long-term efficacy yet. Therefore, we conduct this study to find the effectiveness of wound injection of rAd-p53 in preventing local recurrence of oral tumor after a radical resection.

Depending on the primary tumor site and stage of disease, patients with oral squamous cell carcinomas develop locoregional recurrence in $15-30 \%$ of cases. The residual tumor cells or pre-malignant cells in a resection margin are believed as a high risk for local recurrence. Wide local excision and high-dose radiotherapy were traditional methods for preventing local recurrence. But the sequelae of those methods cause substantial morphological and functional damage. In this study we used the surgical wound surface injection of rAd-p53 combined with radiotherapy to prevent local recurrence after a tumorectomy. Preliminary data presented in this report indicate a trend that this treatment regiment did decrease the local recurrent rate. The data from 
Citation: Liu S, Chen P, Hu M, Tao Y, Chen L, et al. (2012) Phase II Study of Post-surgery Radiotherapy Combined with Recombinant Adeno-viral Human P53 Gene Therapy in Treatment of Oral Cancer. J Cancer Sci Ther 4: 193-195. doi:10.4172/1948-5956.1000140

the long-term follow-up for all the study cases will provide clearer evidence of reducing the local recurrent rate. The main adverse events relative to the rAd-p53 injection were self-limited fever, indicating it is safe for local application of rAd-p53.

The sequelae of surgical resection and high-dose irradiation cause substantial changes in head and neck anatomy, leading to considerable problems in the early morphological detection of recurrent disease.

\section{References}

1. Sakai E, Rikimaru K, Ueda M, Matsumoto Y, Ishii N, et al. (1992) The p53 tumor-suppressor gene and ras oncogene mutations in oral squamous-cell carcinoma. Int J Cancer 52: 867-872.

2. Yamazaki Y, Chiba I, Hirai A, Sugiura C, Notani K, et al. (2003) Specific p53 mutations predict poor prognosis in oral squamous cell carcinoma. Oral Oncol 39: 163-169.

3. Seemann S, Maurici D, Olivier M, Caron de Fromentel C, Hainaut P (2004) The tumor suppressor gene TP53: implications for cancer management and therapy. Crit Rev Clin Lab Sci 41: 551-583.

4. Kirsch DG, Kastan MB (1998) Tumor-suppressor p53: implications for tumor development and prognosis. J Clin Oncol 16: 3158-3168.

5. Pan JJ, Zhang SW, Chen CB, Xiao SW, Sun Y, et al. (2009) Effect of recombinant adenovirus-p53 combined with radiotherapy on long-term prognosis of advanced nasopharyngeal carcinoma. J Clin Oncol 27: 799-804.

6. Gurnani M, Lipari P, Dell J, Shi B, Nielsen LL (1999) Adenovirus-mediated p53 gene therapy has greater efficacy when combined with chemotherapy against human head and neck, ovarian, prostate, and breast cancer. Cancer Chemother Pharmacol 44: 143-151.
7. Yu M, Chen W, Zhang J (2010) p53 gene therapy for pulmonary metastasis tumor from hepatocellular carcinoma. Anticancer Drugs 21: 882-884.

8. Keedy V, Wang W, Schiller J, Chada S, Slovis B, et al. (2008) Phase I study of adenovirus p53 administered by bronchoalveolar lavage in patients with bronchioloalveolar cell lung carcinoma: ECOG 6597. J Clin Oncol 26: 4166 $-4171$

9. Yang ZX, Wang D, Wang G, Zhang QH, Liu JM, et al. (2010) Clinical study of recombinant adenovirus-p53 combined with fractionated stereotactic radiotherapy for hepatocellular carcinoma. J Cancer Res Clin Oncol 136: 625630 .

10. Tian G, Liu J, Zhou JS, Chen W (2009) Multiple hepatic arterial injections of recombinant adenovirus p53 and 5-fluorouracil after transcatheter arteria chemoembolization for unresectable hepatocellular carcinoma: a pilot phase II trial. Anticancer Drugs 20: 389-395

11. Kastan MB, Canman CE, Leonard CJ (1995) P53, cell cycle control and apoptosis: implications for cancer. Cancer Metastasis Rev 14: 3-15.

12. Muller PA, Vousden KH, Norman JC (2011) p53 and its mutants in tumor cell migration and invasion. J Cell Biol 192: 209-218.

13. Weller M (1998) Predicting response to cancer chemotherapy: the role of $p 53$ Cell Tissue Res 292: 435-445.

14. Matsubara H, Kimura M, Sugaya M, Koide Y, Gunji Y, et al. (1999) Expression of wild-type p53 gene confers increased sensitivity to radiation and chemotherapeutic agents in human esophageal carcinoma cells. Int $\mathrm{J}$ Oncol 14: 1081-1085

15. Qi X, Chang Z, Song J, Gao G, Shen Z (2011) Adenovirus-mediated p53 gene therapy reverses resistance of breast cancer cells to adriamycin. Anticancer Drugs 22: $556-562$ 